Population Study

Poster

Abstract ID: 103

\title{
Incidence of chronic Hepatitis B among Orang Asli in Pahang
}

Mohd Al-Baqlish Mohd Firdaus ${ }^{\mathrm{a}} \mid$ Tee Hoi Poh $^{\mathrm{b}}$

${ }^{a}$ Kulliyah of Medicine, International Islamic University Malaysia

${ }^{b}$ Medical Department, Hospital Tengku Ampuan Afzan Pahang Malaysia

Introduction: The rate of Hepatitis B infection in Malaysia is $4.7 \%$ and the prevalence of Hepatitis $B$ infection varies among ethnic group in Malaysia. The estimated prevalence of chronic Hepatitis B among Orang Asli in Malaysia is 8.3\%. Methods: 232 Orang Asli participated in this study on a voluntary basis during two health screening in two Orang Asli Villages. Subjects serum HBsAg and HBsAb titres were taken and analyzed. Results: From 232 participants, there are 24 subjects who are HBsAg positive (10.3\%) and only $15.9 \%$ of the population study has immunity to Hepatitis B. Conclusions: The incidence of chronic Hepatitis B among Orang Asli in Pahang is higher than the general population, and the rate of immunity to Hepatitis $B$ are low compared to the general population.

KEYWORDS: Hepatitis B, Prevelance, Orang Asli, Pahang, Malaysia 